\title{
Methicillin-Resistant Staphylococcus aureus-Associated Diarrhea in a Critically III Burn Patient
}

\author{
Aldin Malkoc ${ }^{\text {a, }}$, David T. Wong ${ }^{\text {b, c }}$
}

\begin{abstract}
Described in surgical patients after antibiotic use in the 1950s and 1960 s, Staphylococcus aureus (S. aureus) enterocolitis is a rare form of nosocomial diarrhea. However, $S$. aureus is not routinely tested like Clostridium difficile ( $C$. difficile). We report a case of methicillinresistant $S$. aureus (MRSA) enterocolitis found on stool culture in a 22-week pregnant female with a previously negative nasal MRSA culture, and a total burn surface area greater than $60 \%$. She also developed necrotizing MRSA pneumonia and bacteremia. After starting broad-spectrum antibiotic for the necrotizing pneumonia with subsequent acute respiratory distress syndrome, the patient exhibited large voluminous diarrhea that tested positive for MRSA and negative for C. difficile in the stool culture. Similar to other reports of high-volume diarrhea, the diarrhea resolved quickly with enteral vancomycin. $S$. aureus should be considered along with $C$. difficile during the workup of nosocomial diarrhea, especially with exposure to broad-spectrum antibiotics in the critically ill patient.
\end{abstract}

Keywords: Enterocolitis; MRSA; Staphylococcus aureus; Nosocomial diarrhea; Vancomycin; Clostridium difficile

\section{Introduction}

Since the mid-1970s, conclusions related to the occurrence of antibiotic-associated enterocolitis (AAE) have been widely associated with Clostridium difficile (C. difficile). In these cases, patients were typically exposed to antibiotics [1]. Discovered in the 1950s, Staphylococcus aureus (S. aureus) was the first well known cause of AAE, but it was soon disputed after the discovery of $C$. difficile. At the time, scientists believed $S$. aureus potentially represented an unrecognized $C$. difficile-relat-

Manuscript submitted February 26, 2021, accepted March 13, 2021

Published online May 13, 2021

aSt. George's University School of Medicine, True Blue Rd, True Blue, Grenada

${ }^{\mathrm{b}}$ Arrowhead Regional Medical Center, 400 N Pepper Ave, Colton, CA 92324, USA

${ }^{\mathrm{c}}$ Corresponding Author: David T. Wong, Arrowhead Regional Medical Center, 400 N Pepper Ave, Colton, CA 92324, USA. Email: davewo@msn.com

doi: https://doi.org/10.14740/jmc3690 ed disease instead [1-3]. While only $15-25 \%$ of today's AAE cases are attributed to $C$. difficile, recognition of $S$. aureus as a causative agent of AAE remains rare, especially in the absence of routine testing. Despite the fact that methicillin-resistant S. aureus (MRSA) is isolated in $55 \%$ of intensive care unit patients in the USA, it is not commonly established in Western literature as a cause of AAE in critically ill patients [4]. However, cases in Australia, Japan, and France have reported MRSA as a well recognized nosocomial pathogen for AAE in hospitalized patients [5]. In this paper, we present a case of enterocolitis caused by MRSA following antibiotic treatment for a patient with necrotizing multilobar pneumonia and $60 \%$ total burn surface area (TBSA).

\section{Case Report}

A 26-year-old pregnant (22 weeks of gestation) female with amphetamine and marijuana use was presented to our Burn Center following a home explosion. This resulted in $60 \%$ TBSA second- and third-degree burn wounds to all extremities circumferentially, bilateral shoulders, neck, and abdomen. Most of the patient's burns were second degree. The patient had a Glasgow Coma Scale of 15 upon arrival to the trauma bay. The patient was hypotensive and tachycardic and was intubated immediately to protect her airway during burn resuscitation. On admission the patient received an MRSA nasal swab that was negative; however, a subsequent MRSA nasal swab on hospital day 3 was positive, as shown in Figure 1.

On physical exam, she was edematous from resuscitation and continued to exhibit normal fetal heart tones. On hospital day 4 , the patient required increasing fraction of inspired oxygen and positive end-expiratory pressure support. The patient was then transitioned to volumetric diffusive respiratory (VDR). Paralytics were required for ventilator asynchrony despite sedation and pain medication as she developed acute respiratory distress syndrome (ARDS). On hospital day 5, the patient showed increasing levels of both lactate and white blood cells. The endotracheal aspiration culture grew MRSA, Enterobacter, and Escherichia coli (E. coli). Additionally, it was noted that the patient was experiencing greater than two loose bowel movements requiring a rectal tube to protect the burn wounds from contamination. The patient was then started on vancomycin and piperacillin/clavulanate intravenously and eventually transitioned to a conventional ventilator.

The patient's hospital course was complicated with an is- 


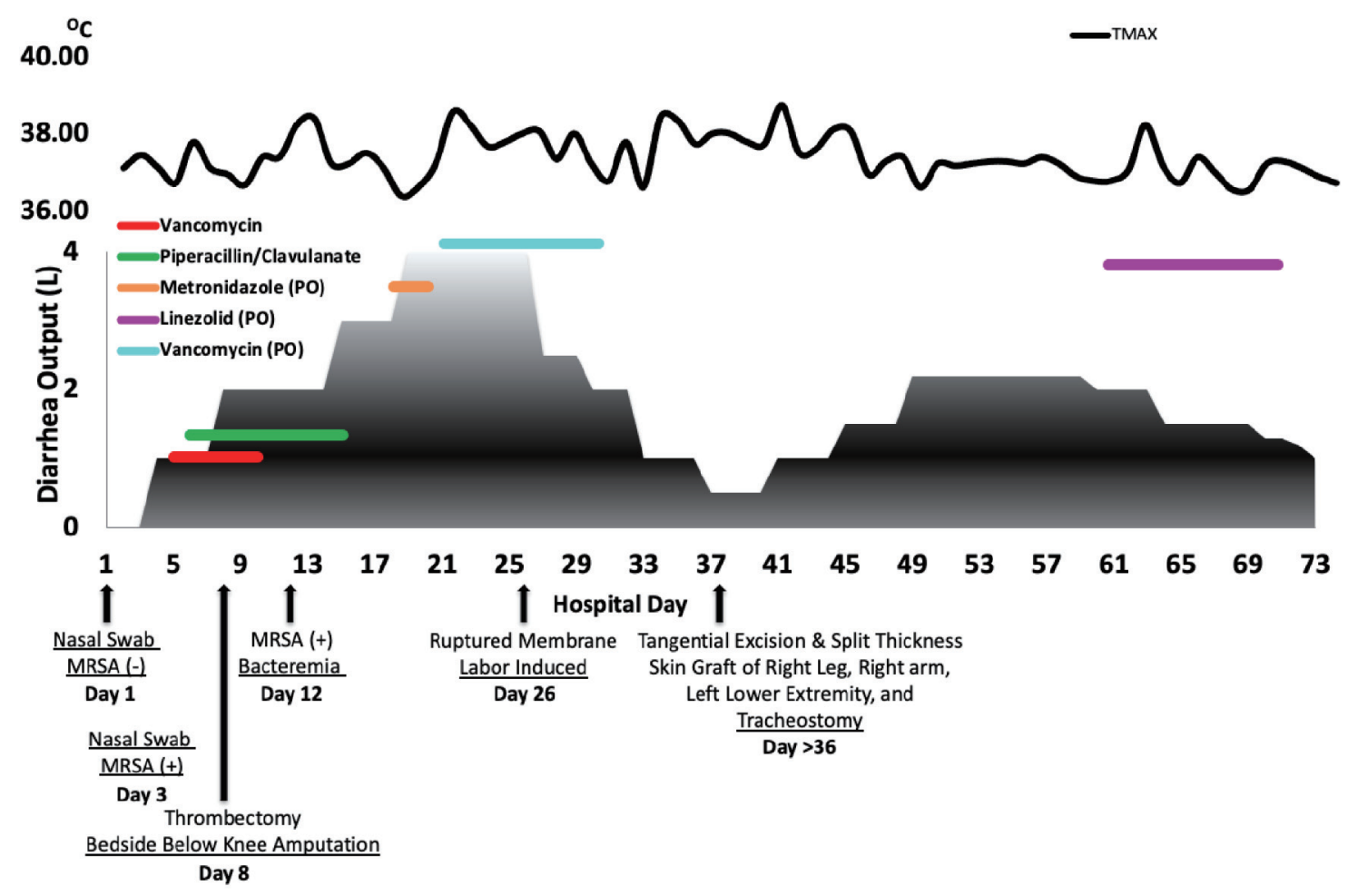

Figure 1. The patient's clinical course focused on the diarrhea output. The patient's various procedures, daily max temperature readings, and antibiotic course treating the patients diarrhea are highlighted in these charts. MRSA: methicillin-resistant $S$. aureus.

chemic right foot on day 8 . The etiology is likely due to the combination of shock, femoral arterial line, as well as preexisting vascular injury due to multiple surgeries in the past for clubfoot. Amputation of the foot was performed when vascular repair was not possible. During this period, the patient's lung injury worsened and again required a VDR (Fig. 1). She continued to experience rising lactate levels with increasing diarrhea volume. On hospital day 12, blood cultures showed MRSA bacteremia. Bronchoscopy was performed and showed tracheitis with loose secretions. The mucosa distal to the carina was normal.

Due to the increasing white blood cells and bands, the team was concerned that the patient may have developed chorioamnionitis and endometritis. Therefore, she was administered gentamicin and clindamycin per obstetrics recommendations. She continued to experience increased stool content and worsening lactate, leading the team to suspect enterocolitis. She also experienced worsening watery diarrhea. At this point, the team suspected pseudomembranous colitis, and metronidazole was added via enteral route. After administering the metronidazole, the patient showed little or no improvement, and stool cultures tested negative for $C$. difficile toxins A and B. The patient was unstable for sigmoidoscopy. Decubitus positioning resulted in severe hypoxia. Ultimately, a stool culture showed MRSA growth, and treatment was converted to enteral vancomycin. Watery diarrhea began to subside within $48 \mathrm{~h}$ of administering enteral vancomycin. At this point, the patient's clinical course was focused on treating her ARDS. She con- tinued to experience worsening hypoxia, requiring VDR and paralysis. Linezolid replaced intravenous vancomycin for better lung penetration. Enteral vancomycin was continued for 10 days, representation of her clinical diarrheal course is shown in Figure 1.

On hospital day 26, it was found that the patient had experienced a rupture of the maternal membrane, Fern positive, which required immediate induction of labor. The fetus was delivered with no spontaneous movements, no spontaneous breathing, and no tone. Rescue efforts were not performed by family request due to the poor prognosis. Following the delivery of the fetus, the patient's condition began to improve. By hospital day 40 the patient showed reduced oxygenation requirements, reduced ventilator support, and improved chest $\mathrm{X}$-ray as shown in Figure 2a-c. She subsequently required skin grafting and was discharged to the acute ventilator rehabilitation facility after 72 days with near complete resolution of lung opacities shown in Figure 2d. The computed tomography (CT) on hospital day 20 demonstrated diffuse bilateral infiltrates, pleural thickening and subpleural cystic changes (Fig. 2e, f) at that time.

\section{Discussion}

MRSA or non-MRSA is a serious nosocomial and communityacquired pathogen. Even though it is well established, there is little research to highlight its cause of enterocolitis. In the early 

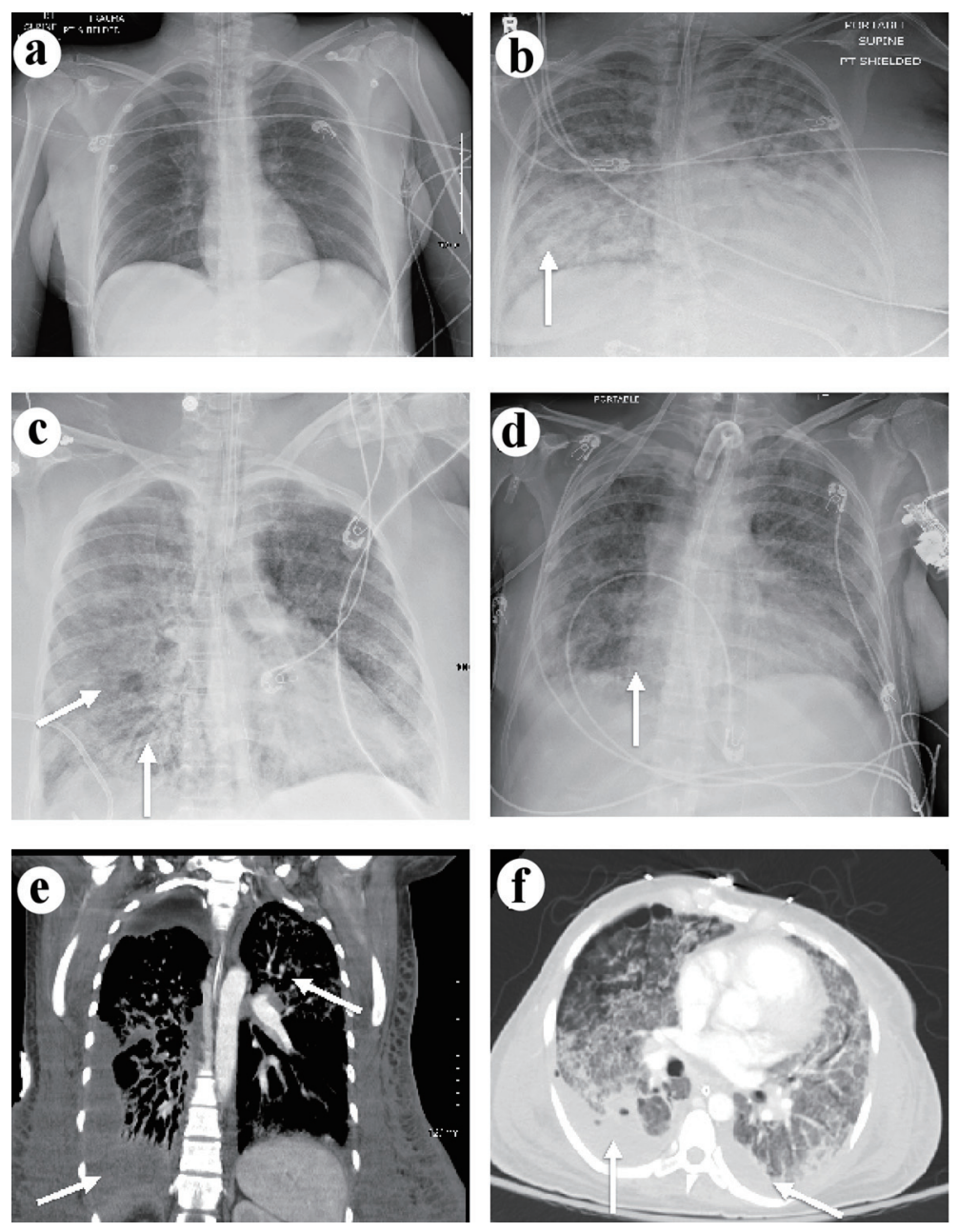

Figure 2. (a) Chest X-ray of the lungs on admission demonstrating normal lung space. (b) Chest X-ray of the lungs on hospital day 20 showing lung opacities on the right side indicated by the arrow. (c) Chest X-ray of the lungs on hospital day 40 showing improving lung opacities indicated by the arrows. (d) Chest X-ray of the lungs on hospital day 72 showing near resolution of lung opacities indicated by the arrow. (e) Coronal CT on hospital day 20 of the lungs demonstrating the diffuse bilateral infiltrates more prominent on the right side indicated by the arrows. (f) Axial CT on hospital day 20 of the lungs demonstrating pleural thickening and diffuse bilateral infiltrates with subpleural cystic changes indicated by the arrows. CT: computed tomography.

1950s and 1960s, S. aureus was thought to cause the majority of AAE and enterocolitis cases in patients with predisposing factors [3]. However, as $C$. difficile cytotoxin became implicated as the major cause of pseudomembranous colitis with routine testing, the appreciation for $S$. aureus declined [6]. Furthermore, there are many other rare causes of pseudomembranous colitis that likely go unrecognized such as Clostridium perfringens, Klebsiella, E. coli O157:H7, and Salmonella enterica (S. enterica) [7,8]. While cases in other countries have reported MRSA as a well recognized nosocomial pathogen for antibiotic-associated enterocolitis in hospitalized patients, North America has seen relatively few MRSA colitis cases $[5,9]$. However, this is likely due to the fact that physicians often do not order stool cultures for hospitalized patients. In addition, enteral vancomycin used empirically for $C$. difficile would have also treated $S$. aureus. With resolution of the diarrhea, clinicians would forego further studies resulting in under reporting.
Similar to $C$. difficile, the infection risk factors associated with MRSA enterocolitis include advanced age, immunosuppression, a prolonged hospital stay, previous antibiotic treatment, and previous $S$. aureus colonization $[9,10]$. In this case, the large burns and subsequent profound systemic inflammatory response syndrome compromised her immune system [11]. This combination likely caused the patient to develop necrotizing MRSA pneumonia and enterocolitis. We suspect this patient was an MRSA carrier. Even though the nasal MRSA was negative on admission, current studies suggest that surveillance via the anterior nares is not sufficient to identify a patient with MRSA. Instead, either repeat testing or stool testing provides a more accurate approach [12].

This case utilized the definition of diarrhea from Boyce et al, which involves three or more loose stools, or greater than $250 \mathrm{~mL}$ of liquid stool, from the rectum as characterized by a rectal tube per $24 \mathrm{~h}$ for greater than 2 days. Additionally, nosocomial diarrhea was classified as an onset of diarrhea oc- 
curring 3 or more days after hospital admission [4]. As demonstrated in other studies, MRSA diarrhea was classified as MRSA growth on stool culture, with absent or scant normal aerobic gram-negative enteric flora, ova, and parasites, negative $C$. difficile toxin in stool, and no possible attribution to medication $[2,4]$. Our patient met the criteria for nosocomial diarrhea with initial stool cultures that showed MRSA growth with no other pathogens.

As noted in existing literature, stool leukocytes are often negative in cases of $S$. aureus enterocolitis and tests are typically not ordered [10]. We suspect that stool leukocytes are often diluted in cases of high-volume diarrhea and do not serve as an accurate method of screening for $S$. aureus enterocolitis. When the patient initially developed large-volume diarrhea, we elected to only order a $S$. aureus stool culture and not stool leukocytes. The clinical presentation along with the negative C. difficile toxin antigens prompted us to treat the patient empirically using enteral vancomycin. Similar to other studies, we treated the patient with $250 \mathrm{mg}$ of enteral vancomycin every $6 \mathrm{~h}$ for 10 days $[1,12]$. Within the first $48 \mathrm{~h}$ the patient showed clinical improvement with reduced watery diarrhea. Rapid response and positive MRSA stool culture strongly suggest $S$. aureus as the causative agent, especially when other treatments failed.

\section{Conclusions}

Hospital-acquired diarrhea is unfortunately a common occurrence that is not always caused by $C$. difficile. Although $C$. difficile is one of the most common infectious causes of antibiotic-associated diarrhea, $S$. aureus is a clinically relevant and under-diagnosed etiology. While our patient's enterocolitis was from MRSA, it is important to note that the literature reports that non-MRSA can also cause enterocolitis [13]. The treatment is the same. Like our patient, the literature reports similar response to enteral vancomycin with improvement within $48 \mathrm{~h}$. Enterocolitis in the critically ill is life-threatening. Other causes of high-volume diarrhea should be sought in symptomatic patients who test negative for $C$. difficile toxins.

\section{Acknowledgments}

The authors would like to thank and express their gratitude to the Edward G. Hirschman Burn Center and the exceptional nursing staff and unit managers for their expert clinical support.

\section{Financial Disclosure}

The authors have no financial or funding disclosures.

\section{Conflict of Interest}

The authors declare there is no conflict of interest.

\section{Informed Consent}

Informed consent was obtained from the family of the patient. The patient was also appropriately de-identified for this manuscript.

\section{Author Contributions}

AM performed the initial manuscript write-up, literature review, and editing of the manuscript. DTW attended on the case and contributed to decision-making, management of the patient, literature review, as well as editing of the manuscript.

\section{Data Availability}

The authors declare that data supporting the findings of this study are available within the article.

\section{Abbreviations}

AAE: antibiotic-associated enterocolitis; $C$. difficile: Clostridium difficile; MRSA: methicillin-resistant $S$. aureus; TBSA: total burn surface area; VDR: volumetric diffusive respiratory; ARDS: acute respiratory distress syndrome

\section{References}

1. Lin Z, Kotler DP, Schlievert PM, Sordillo EM. Staphylococcal enterocolitis: forgotten but not gone? Dig Dis Sci. 2010;55(5):1200-1207.

2. Avery LM, Zempel M, Weiss E. Case of antibiotic-associated diarrhea caused by Staphylococcus aureus enterocolitis. Am J Health Syst Pharm. 2015;72(11):943-951.

3. Pressly KB, Hill E, Shah KJ. Pseudomembranous colitis secondary to methicillin-resistant Staphylococcus aureus (MRSA). BMJ Case Rep. 2016;2016:bcr-2016-215225.

4. Boyce JM, Havill NL. Nosocomial antibiotic-associated diarrhea associated with enterotoxin-producing strains of methicillin-resistant Staphylococcus aureus. Am J Gastroenterol. 2005;100(8):1828-1834.

5. Boyce JM, Havill NL, Maria B. Frequency and possible infection control implications of gastrointestinal colonization with methicillin-resistant Staphylococcus aureus. J Clin Microbiol. 2005;43(12):5992-5995.

6. Froberg MK, Palavecino E, Dykoski R, Gerding DN, Peterson LR, Johnson S. Staphylococcus aureus and Clostridium difficile cause distinct pseudomembranous intestinal diseases. Clin Infect Dis. 2004;39(5):747-750.

7. Polage CR, Solnick JV, Cohen SH. Nosocomial diarrhea: evaluation and treatment of causes other than Clostridium difficile. Clin Infect Dis. 2012;55(7):982-989.

8. Kennedy J, Simmonds L, Orme R, Doherty W. An unusual case of Escherichia coli O157:H7 infection with pseudomembranous colitis-like lesions associated with 
haemolytic-uraemic syndrome and neurological sequelae. BMJ Case Rep. 2017;2017:bcr-2016-218586.

9. Bergevin M, Marion A, Farber D, Golding GR, Levesque S. Severe MRSA enterocolitis caused by a strain harboring enterotoxins D, G, and I. Emerg Infect Dis. 2017;23(5):865-867.

10. Lane AB, Copeland NK, Onmus-Leone F, Lawler JV. Methicillin-Resistant Staphylococcus aureus as a probable cause of antibiotic-associated enterocolitis. Case Rep Infect Dis. 2018;2018:3106305.

11. Townsend CM, Beauchamp RD, Evers BM, Mattox KL. Sabiston textbook of surgery E-book. Elsevier Health
Sciences; 2016.

12. Sizemore EN, Rivas KM, Valdes J, Caballero J. Enteral vancomycin and probiotic use for methicillin-resistant Staphylococcus aureus antibiotic-associated diarrhoea. BMJ Case Rep. 2012;2012:bcr-2012-006366.

13. Lemyre B, Xiu W, Bouali NR, Brintnell J, Janigan JA, Suh KN, Barrowman N. A decrease in the number of cases of necrotizing enterocolitis associated with the enhancement of infection prevention and control measures during a Staphylococcus aureus outbreak in a neonatal intensive care unit. Infect Control Hosp Epidemiol. 2012;33(1):2933. 